\title{
1 Regionalisation of population growth projections in coastal exposure analysis
}

Jan-Ludolf Merkens $\mathrm{a}^{\mathrm{a}^{*}}$, Daniel Lincke ${ }^{\mathrm{b}}$, Jochen Hinkel ${ }^{\mathrm{b}, \mathrm{c}}$, Sally Brown ${ }^{\mathrm{d}, \mathrm{e}}$, Athanasios Thomas Vafeidis ${ }^{\mathrm{a}}$

${ }^{a}$ Department of Geography, Kiel University, Ludewig-Meyn-Str. 14, 24118 Kiel, Germany

${ }^{b}$ Global Climate Forum e.V. (GCF), Neue Promenade 6, 10178 Berlin, Germany

${ }^{c}$ Division of Resource Economics, Albrecht Daniel Thaer-Institute and Berlin Workshop in Institutional Analysis of Social-Ecological Systems (WINS), Humboldt-University, Berlin

${ }^{d}$ Faculty of Engineering and the Environment, University of Southampton, University Rd, Southampton SO17 $1 B J, U K$

${ }^{e}$ Tyndall Centre for Climate Change Research, Southampton, UK

* merkens@geographie.uni-kiel.de; FON +49 431 880-1701; FAX +49431 880-4658

\section{Abstract}

Large-area coastal exposure and impact analysis has focussed on using sea-level rise (SLR) scenarios and has placed little emphasis on socioeconomic scenarios, while neglecting spatial variations of population dynamics. We use the Dynamic Interactive Vulnerability Assessment (DIVA) Framework to assess the population exposed to 1 in 100-year coastal flood events under different population scenarios, that are consistent with the Shared Socioeconomic Pathways (SSPs); and different SLR scenarios, derived from the Representative Concentration Pathways (RCPs); and analyse the effect of accounting for regionalised population dynamics on population exposure until 2100. In a reference approach, we use homogeneous population growth on national level. In the regionalisation approaches, we test existing spatially explicit projections that also account for urbanisation, coastal migration and urban sprawl. Our results show that projected global exposure in 2100 ranges from 100 million to 260 million, depending on the combination of SLR and population scenarios and method used for regionalising the population projections. The assessed exposure based on the regionalised approaches is higher than that derived from the reference approach by up to 60 million people (39\%). Accounting for urbanisation and coastal migration leads to an increase in exposure, whereas considering urban sprawl leads to lower exposure. Differences between the reference and the regionalised approaches increase with higher SLR. The regionalised approaches show highest exposure under SSP5 over most of the $21^{\text {st }}$ century, although total population in SSP5 is the second lowest overall. All methods project the largest absolute growth in exposure for Asia and relative growth for Africa.

Keywords: sea-level rise, Shared Socioeconomic Pathways, coastal population dynamics, coastal flooding exposure 


\section{Introduction}

A large number of studies have assessed future coastal exposure to sea-level rise (SLR) and respective impacts on global scale (e.g. Hanson et al. 2011; Hallegatte et al. 2013; Neumann et al. 2015). These studies rely on SLR and socio-economic scenarios, because future climate and socio-economic change cannot be forecasted over decades due to deep uncertainties and alternating pathways of development involved. While a lot of emphasis has been placed on developing adequate SLR scenarios that account for uncertainties in future SLR, much less emphasis has been placed on socio-economic scenarios, even though both uncertainties are roughly at equal footing in terms of their influence on future coastal exposure and impacts (Hinkel et al. 2014).

The implementation of population changes in global coastal impact assessments has generally improved since the 1990s, as at that time studies assumed socioeconomic conditions to remain constant (e.g. Nicholls and Mimura 1998) and were therefore unrealistic for future conditions. In recent years multiple scenarios of socioeconomic development on global, continental or national level have been employed in global coastal impact assessment in order to account for uncertainties in socioeconomic development and lead to plausible estimates on future exposure (see e.g. Nicholls (2004) and Arnell et al. (2004) for the Intergovernmental Panel on Climate Change (IPCC) Special Report on Emission Scenarios (SRES) and e.g. Hinkel et al. (2014) for the Shared Socioeconomic Pathways (SSPs)). However, these approaches used population projections on national level and did not account for different population change rates in coastal and inland areas. As coastal zones typically face different challenges compared to inland areas, including differing rates of economic growth and a higher density of cities (McGranahan et al. 2007; Seto 2011; Kummu et al. 2016), coastal population was underestimated.

For this reason, some recent studies of global coastal exposure have used higher growth rates for coastal population than for inland population. Nicholls et al. (2008) assumed coastal population to grow up to 2 times faster than the national average. Neumann et al. (2015) refined the approach of Nicholls et al. (2008) and differentiated between coastal and inland population development for urban and non-urban areas by using correction factors. These corrections factors allowed coastal population to remain constant if inland population was projected to decrease and grew 1.7 to 2 times faster than the inland population if the inland population was projected to increase. These approaches have the limitations of assuming first, arbitrary correction factors, and second that coastal population develop faster than inland population for all countries, which is, not always the case. Merkens et al. (2016), for example, tested this assumption against historical population data for coastal countries between 1990 and 2000 and found that for $40-50 \%$ of all countries inland urban and rural locations grow faster than their coastal counterparts. 
Spatially explicit population projections provide a more realistic basis for coastal exposure analysis. Gaffin et al. (2004) developed population projections until 2100 consistent with the SRES with a horizontal resolution of 15, ( $\sim 30 \mathrm{~km}$ at the equator). Grübler et al. (2007) produced gridded population projections with a horizontal resolution of $7.5^{\prime}(\sim 15 \mathrm{~km}$ at the equator) for three of four SRES scenarios. Their work was refined by Jones and O’Neill (2016), who created gridded population projection for all five SSPs at an initial horizontal resolution of 7.5'. Their projections were downscaled to $0.5^{\prime}(\sim 1 \mathrm{~km}$ at the equator) by Gao (2017). Jones and O'Neill (2016) analysed historical trends of population development and used a gravity-based downscaling model to simulate urban and rural population changes. For all five SSPs an index of potential attractiveness for each grid cell was used to allocate population, which indirectly leads to different growth rates on subnational level for coastal and inland areas. Merkens et al. (2016) created gridded population projections with a horizontal resolution of $0.5^{\prime}$ for all five SSPs that focused on coastal areas and analysed historical growth differences of coastal urban and coastal rural areas compared to the inland counterparts. Their method is described in more detail in section 2.2. In addition, they expanded the qualitative narratives of the SSPs to the coastal zone and assumed scenario-specific modifications of the observed growth differences that are based on the narratives. Both studies, Jones and O'Neill (2016) and Merkens et al. (2016), are consistent with the population projections (KC and Lutz 2017) and urbanisation projections (Jiang and O'Neill 2017) on national level that are used in the SSP framework (O'Neill et al. 2017).

In this study we assess the sensitivity of outcomes in coastal exposure analysis to inclusion of subnational heterogeneity in population projections. We compare (i) homogeneous population change on national level (hereinafter referred to as the basic approach) to (ii) the population projections of Merkens et al. (2016) that also account for urbanisation and coastal migration are have been specifically developed for coastal exposure analysis (hereinafter referred to as the coastal approach) and to (iii) the downscaled spatial projections of Jones and O'Neill (2016) by Gao (2017) that account for urbanisation and urban sprawl (referred to as dynamic approach)). We further analyse (iv) the extent to which urbanisation can explain the differences in exposure between the basic and coastal approach (referred to as urban approach) (see Fig. 1). 
BASIC APPROACH

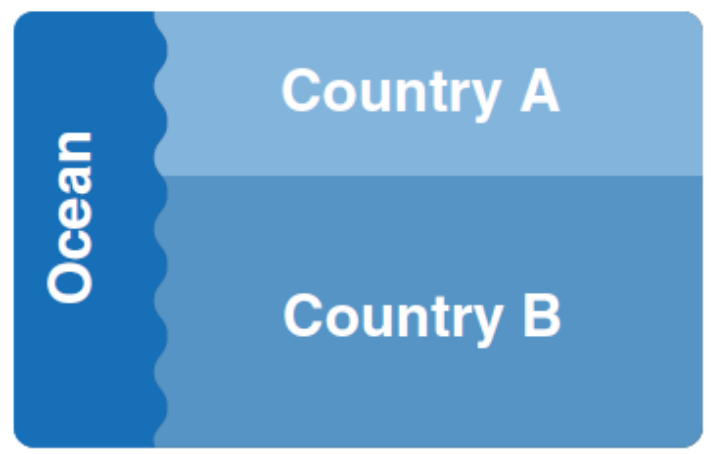

DYNAMIC APPROACH

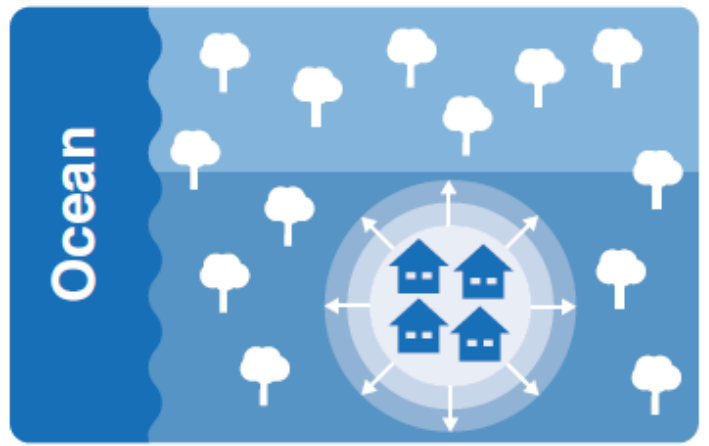

COASTAL APPROACH

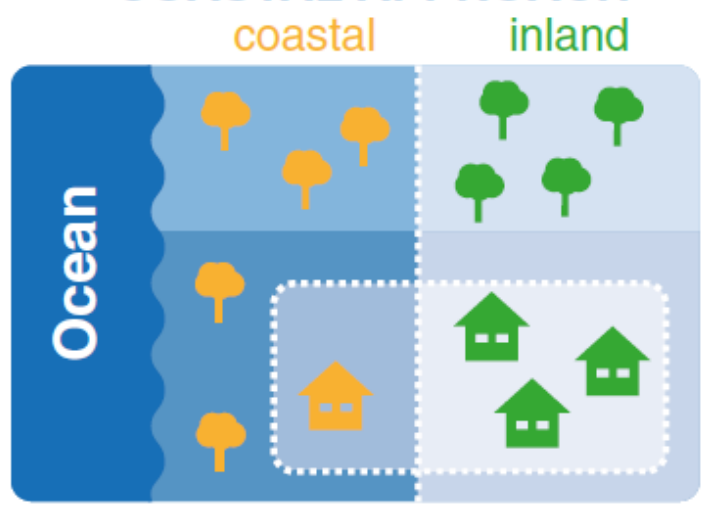

URBAN APPROACH

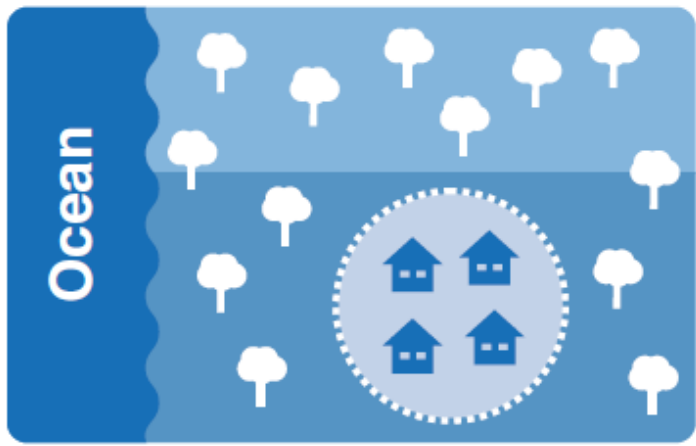

Fig. 1: Regionalisation approaches. The basic approach assumes homogeneous population dynamics within a country. The coastal approach differentiates population dynamics between coastal urban, coastal rural, inland urban and inland rural areas. The dynamic approach uses dynamic urban extents to account for urban sprawl. The urban approach differentiates urban and rural population dynamics with static urban extents

\section{Data and Methods}

\subsection{DIVA database}

For our analysis, we employ the Dynamic Interactive Vulnerability Assessment (DIVA) modelling framework, which has been used in a wide range of applications in coastal risk assessments (see Hinkel et al. 2013 for erosion, Hinkel et al. 2010 and Hinkel et al. 2014 for adaptation, Hinkel et al. 2012 for adaptation and mitigation, Spencer et al. 2016 for wetlands). The results presented in this study are based on version 30 of the DIVA database and model version 1.7

The DIVA database breaks the world's coasts (excluding Antarctica) into 12,148 segments. Each coastal segment provides information on administrative, bio-physical and socioecological attributes. In the context of this study, we focus on the population living in the 1 in 100-year floodplain, which is a well-established measure of coastal exposure analysis (e.g. Hanson et al. 2011; Vousdoukas et al. 2016; Muis et al. 2017). The 1 in 100-year coastal flood heights are taken from Muis et al. (2016). We use the DIVA flood module to calculate the number of people 
living in the floodplain without considering dikes. A detailed description of this approach can be found in Hinkel et al. (2014). As we account for isostatic adjustment and subsidence (see section 2.3), DIVA provides relative sealevel for all segments.

To define the floodplain, we use a global elevation dataset which is based on SRTM (Jarvis et al. 2008) and GTOPO30 (USGS 1996) data for high latitudes $\left(>60^{\circ} \mathrm{N}\right.$ and $\left.>54^{\circ} \mathrm{S}\right)$. For all elevation steps from $1 \mathrm{~m}$ to $16 \mathrm{~m}$, we calculate the extent of the area that is hydrologically connected to the ocean and smaller or equal to the respective elevation threshold (see Poulter and Halpin 2008). Intermediate values are linearly interpolated (Hinkel et al. 2014). We utilise the GRUMPv1 grid (CIESIN et al. 2011a) to analyse the population located in each of these elevation increments for the year 2000. The coastal SSPs of Merkens et al. (2016) use the GRUMP urban extent grid, which uses census population counts, settlement points and night-time lights, to define urban areas (CIESIN et al. 2011b) and assumes assume these to be static. GRUMP tends to underestimate the extent of settlements with none or little light at night, e.g. in parts of Africa (Balk et al. 2006), which also affects the estimates on exposed population. The estimates on exposed population also depend on the elevation model used for the analysis. Lichter et al. (2011) analysed the land area of the LECZ derived from three different elevation datasets with the same vertical and horizontal resolution of $1 \mathrm{~m}$ and $0.5^{\prime}(\sim 1 \mathrm{~km}$ at the equator). On continental scale, they found differences of up to $40 \%$. In the same study, Lichter et al. (2011) compared two commonly used population datasets (GRUMP alpha and LandScan 2006) and analysed the population located in the LECZ. On global scale, the LECZ population differed by $\sim 10 \%$, on continental scale by up to $28 \%$. They stated the combined uncertainty of elevation and population data at $20 \%$ on global scale and at up to $67 \%$ on continental scale. Mondal and Tatem (2012) compared the LECZ population for GRUMP version 1 (the same version that was used in this study) and LandScan 2008 and found differences of $4 \%$ on global scale and of up to $39 \%$ on continental scale. GRUMP's underlying assumption of homogeneous population distribution within urban and rural areas in the same administrative unit can in addition lead to an over- or underestimation of the 'true' exposure (Merkens and Vafeidis 2018). As this study uses the same population and elevation datasets throughout the analysis, we expect the relative differences between the approaches to be independent from the elevation or population data, whereas the absolute numbers are likely to be different if other population or elevation data are used.

\subsection{Socioeconomic scenarios}

We initially calculate exposure of population based on two approaches to account for future population development in coastal areas (see Fig. 1). In the basic approach, we use national population projections taken from KC and Lutz (2017) and apply these to the baseline (i.e., year 2000) spatial population data. This approach assumes 
homogeneous growth rates within each country, i.e. population in coastal areas grows at the same rate than in inland areas. In the coastal approach, we use the coastal SSPs of Merkens et al. (2016). These are based on the national population projections of KC and Lutz (2017) as well, but consider urbanisation projections (Jiang and O’Neill 2017), historical growth differences and scenario-dependent modifications of growth differences. For each country Merkens et al (2016) analysed the population growth for coastal urban (rural) areas and inland urban (rural) areas over a 10-year period from 1990 to 2000. If coastal areas had a higher population growth rate than inland areas, the growth difference (GD) was positive and vice versa. The GD allows for negative (positive) population growth in the coast or inland even if national population growth is positive (negative). It also allows for higher population change rates in coastal areas compared to inland areas. For SSP2 Merkens et al. (2016) assumed the GD to keep constant over time for each location. For the other four SSPs they modified the GDs based on the interpretation of the coastal SSP narratives, which are introduced in the same study. They quantified the modification of the GDs based on the difference between percentiles in the distribution of the observed urban and rural GDs for all coastal countries. In SSP1 they assume no differences in growth for coastal and inland urban areas and a reduced rural GD (translates to relatively higher rural growth in inland). In SSP3 they assume that the GD to reduce by $50 \%$ for both, urban and rural areas. In SSP4 and SSP5 they increased the GD (translates to relatively higher relative growth at the coast), whereby the increase was bigger in SSP5. Based on the scenario specific GDs and the population and urbanisation projections they calculated population counts for coastal urban, costal rural, inland urban and inland rural for each country in 5 year increments until 2100. This leads to heterogeneous growth rates within countries because urban areas develop differently to rural areas and coastal areas differently to inland areas. We then calculate the mean coastal population growth rate for each country and apply it on each coastline segment of this country. We must note that the definitions of 'urban' between GRUMP (used in Merkens et al. (2016) and Gao (2017)) and Jiang and O'Neill (2016) differ, which results in an offset in the data for the years 2005 and 2010 (see section 4 for a discussion of the implications on exposure analysis).

\subsection{Sea-level rise scenarios}

We use the projected changes in global mean sea level and the likely ranges reported in the Fifth Assessment Report of the Intergovernmental Panel on Climate Change (Church et al. 2013). For each of the four RCPs, we use the ensemble median as medium SLR scenario. The $83^{\text {rd }}$ percentile serves as high SLR scenario and the $17^{\text {th }}$ percentile as low SLR scenario (see Table 1). We do not consider regional patterns of SLR due to ocean dynamics and regionally differential changes in thermal expansion and rotational and gravitational effects of the mass loss of ice sheet. Church et al. (2013) show that these regional effects are below $10 \%$ for most of the populated coastal zone with the exception of the East Coast of the US. Hence the global effects of these regional SLR variations are 
expected to be much smaller than those of human-induced subsidence in densely populated river deltas, which we consider here together with isostatic adjustment. Furthermore, uncertainties in regional sea level projections are large, with different models producing different patterns and the highest deviations of regional sea-level rise due to dynamic variability coinciding with those regions for which model uncertainties are largest (Church et al., 2013). We assume that water levels during coastal floods increase by the same amount as the projected global sea-level and do not account for non-linear interactions between the water level and SLR (Arns et al. 2017) as the focus of this paper is the comparison of population distribution approaches.

Table 1: Sea level rise projections for 2100 referenced to the 1986-2005 period [in $\mathrm{m}$ ].

\begin{tabular}{l|lll} 
& low & medium & high \\
\hline RCP2.6 & 0.28 & 0.44 & 0.61 \\
RCP4.5 & 0.36 & 0.53 & 0.71 \\
RCP6.0 & 0.38 & 0.55 & 0.73 \\
RCP8.5 & 0.53 & 0.74 & 0.98 \\
\hline
\end{tabular}

Values are taken from Prather et al. (2014).

In this study, we use the 12 SLR scenarios from Table 1 (four RCPs, for each high, medium and low SLR projections). These are combined with the five SSPs. Taking into account the two regionalisation approaches (plus another two for testing our assumption) in each SSP, we end up with 240 model runs. This number could be reduced by ignoring scenario combinations that are not plausible. For example, the combination of an environmentally friendly socioeconomic scenario (SSP1) and a physical scenario with high radiative forcing (RCP8.5) would in general be inconsistent (van Vuuren et al. 2014; Engström et al. 2016). Nevertheless, we decided to analyse all scenario combinations, as this study aims to analyse and understand the effect that regionalisation approaches of socioeconomic scenarios have for impact assessment.

\section{Results}

We compare future coastal exposure to 1 in 100-year coastal floods based on the different regionalisation approaches. We define the absolute difference in exposure as the difference in the tested approach (i.e. coastal, urban or dynamic) minus the exposure in the basic approach. The relative difference is defined as the absolute difference in exposure divided by the exposure in the basic approach.

\subsection{Global}

Our first main finding is that accounting for urbanisation and coastal migration has significant implications for assessing coastal exposure. The exposure based on the coastal approach exceeds the one based on the basic approach in all scenarios over the $21^{\text {st }}$ century (see Fig. 2). This finding is consistent for all SLR scenarios (see 
Fig. A. 3). For SSP1, 4 and 5 we find the exposure in the basic approach with high SLR in all RCPs to be lower than the respective low SLR variant in the coastal approach. In other words, in these scenarios the difference between the population distribution approaches is larger than the difference between high and low SLR. To investigate which of the two (urbanisation and coastal migration) is the dominant process leading to the difference between basic and coastal approach, we added the 'urban approach' to our modelling scheme (see Fig. 1). The urban approach is based on population and urbanisation projections that are modelled in the same way as in the coastal SSPs, but uses a GD of zero, which means that the population in urban and rural zones for each SSP grows at rates consistent with projections on national level and does not differ between coastal and inland areas. We assume that the difference between the urban approach and the basic approach represents the impact of changing urbanisation levels, without considering urban sprawl. The difference between the urban approach and the coastal approach can result from differences in fertility, mortality, international migration or internal migration, of which we assume internal migration from or to the coast to have the highest impact. We find that, independently of SLR, urbanisation explains $61 \%$ of the difference between the coastal and basic approach in SSP1, 96\% in SSP2, 54\% in SSP3, 76\% in SSP4 and 45\% in SSP5 (see Fig A. 2). This means that SSP5 is the only scenario where urbanisation appears not to be the dominant process. This can be explained by the underlying assumptions of intense coastward migration for SSP5 in the coastal approach (Merkens et al., 2016). In general, the projected increase in urbanisation levels leads to higher population growth rates in the coastal zone compared to inland areas, as coastal areas show a higher density of cities than inland areas, and population is projected to move into these cities. In the basic approach, the population in all areas within a country grows at the same rate, which leads to lower population numbers at the coast compared to the coastal approach. We therefore conclude that the higher exposure in the coastal approach compared to the basic approach is due to a combination of increasing urbanisation levels in all SSPs and migration to coastal areas, of which urbanisation is the dominant process for SSPs 1-4 and coastal migration for SSP5. 


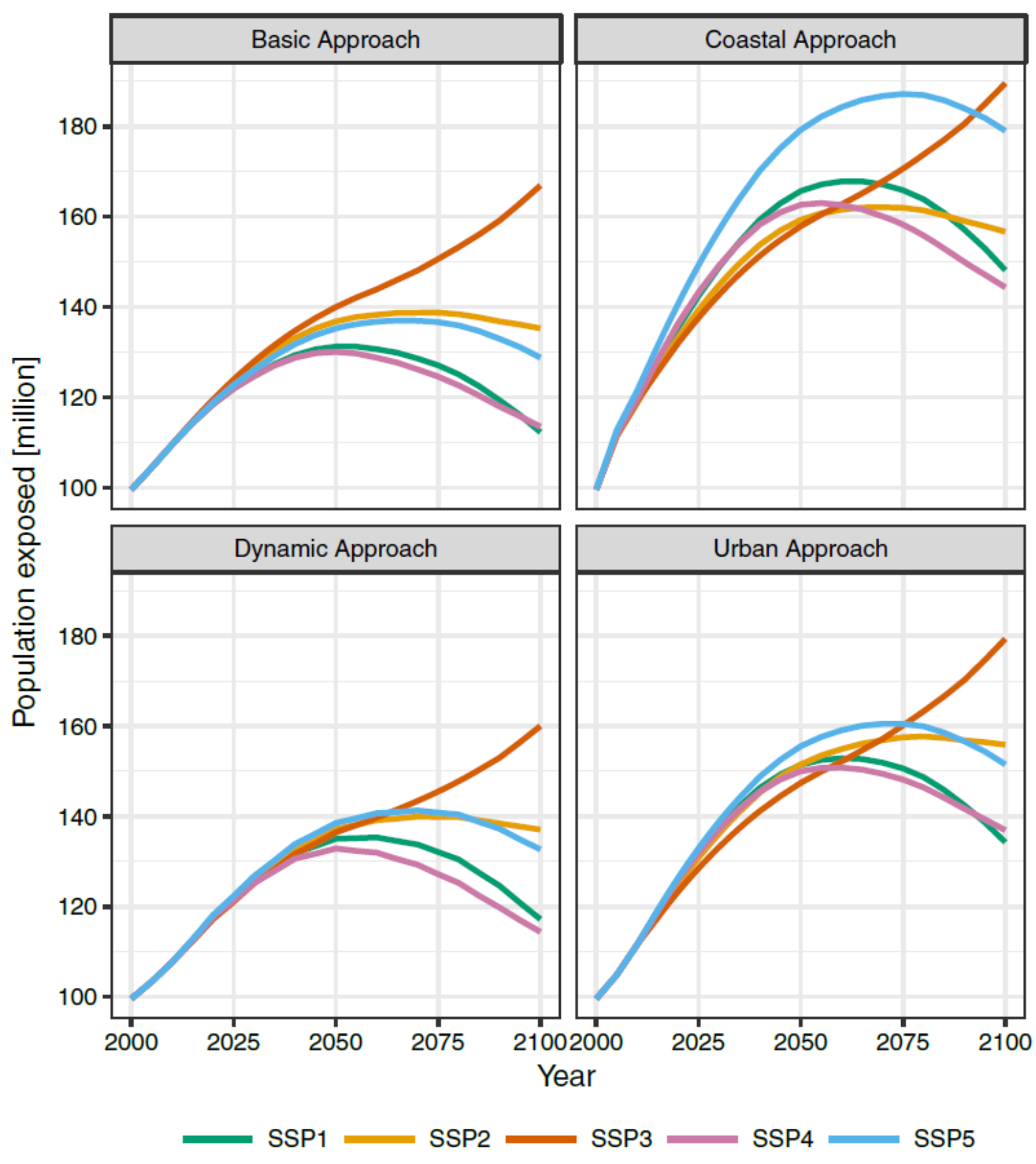

214 Fig. 2: Exposure of population to 1 in 100-year coastal floods under medium SLR in RCP6.0 in the tested approaches.

215 Our second main finding is that the implementation of urban sprawl has a considerable impact on the estimates on 216 exposure. We compare the urban approach to a 'dynamic approach', which is based on the population projections 217 of Jones and O’Neill (2016) that were downscaled by Gao (2017). Unlike the urban (and coastal) approach, that 218 assume urban extent to be static, the dynamic approach considers urban sprawl, which leads to wider city extents and lower population densities within cities. We assume that differences between the urban and dynamic approach are mainly due to urban sprawl, as the approaches use the same population projections of KC and Lutz (2017) and the same urbanisation projections of Jiang and O'Neill (2017). Compared to the dynamic approach, we find exposure to 1 in 100-year coastal floods to be higher in the urban approach for all combinations of SSPs and RCPs 
(Fig. A. 3). They differ between 15 million in SSP1 (RCP 2.6 and low SLR) and 26 million in SSP4 (RCP 8.5 and

high SLR). Differences in SSP1 are lowest, as cities in the dynamic approach are assumed to be concentrated (Jones and O'Neill 2016) and urban extents to be static in the urban (and coastal) approach. However, the difference of 15 million in SSP1 is considerable and suggests that the definition of urban areas (and population) between the urban and the dynamic approach differs, as urbanisation levels and total population do not differ and cities are assumed to be concentrated (dynamic approach) or static (urban approach). For SSPs 2-5 differences between the urban approach and dynamic approach are higher, as only the dynamic approach considers urban sprawl. This suggests that urban sprawl can lead to a reduction of exposure as cities seem to expand towards less flood-prone areas. The differences between the basic and the dynamic approach are rather small (Fig. 2). Global exposure in the dynamic approach under SSP3 for 2100 is up to 7.5 million lower than one in the basic approach. In the other SSPs, exposure based on the dynamic approach exceeds the basic approach by 1 million in SSP4, 2 million in SSP2, 5 million in SSP5 and 6 million in SSP1 (see Fig. A. 4). These SSPs are also projected to have a high increase in urbanisation levels, whereas urbanisation levels in SSP3 are projected to increase little (Jiang and O’Neill 2017). This supports our first finding that neglecting urbanisation patterns would lead to an underestimation of coastal exposure. The differences between the dynamic and the coastal approach are larger than the differences between the dynamic and the urban approach (between 17 million is SSP2 under RCP 2.6 with low SLR and 54 million in SSP5 under RCP 8.5 and high SLR), as coastal migration is additionally considered in the coastal approach. Overall we believe that the coastal approach overestimates exposure, as it does not consider urban sprawl, which appears to reduce exposure; and that the dynamic approach underestimates exposure, as it does not explicitly consider coastal migration, which appears to increase exposure to coastal flooding. We must note that this study does not aim to test the underlying quantifications on coastal migration in Merkens et al. (2016) and the quantification of urban sprawl in Jones and O'Neill (2016), but rather to investigate the implications for coastal exposure analysis when accounting or neglecting of processes actually taking place in coastal areas.

We also find that the population distribution approach is important in determining which SSP leads to the highest exposure to coastal flooding. Though all approaches agree on SSP3 having the highest exposure in 2100, only the basic approach shows SSP3 to lead to the highest exposure throughout the century. The other approaches agree on SSP5 leading to the highest exposure until 2060 (dynamic approach), 2075 (urban approach) and 2090 (coastal approach) (see Fig. 2). This holds true for all SLR scenarios. This is noteworthy as SSP5 and SSP1 are projected to have considerably lower total populations than the other SSPs (KC and Lutz 2017). We identify two factors leading to this observation. The behaviour in the basic approach can be explained by the underlying global population projections that project population to be highest in SSP3 (KC and Lutz 2017). The higher exposure in 
SSP5 in the other approaches is due to high urbanisation levels (Jiang and O'Neill 2017). Exposure rises in the coastal approach as coastal areas are assumed to be more attractive than inland areas and decreases in the dynamic approach as high urban sprawl leads to cities expanding to flood proof areas.

Results also show that the absolute difference in exposed population between the basic and the other approaches increases with SLR (see Fig. A. 4). We find the highest differences under the high SLR projections in RCP8.5 and the smallest differences under the low SLR projections in RCP2.6. Compared to the basic approach, SSP1, SSP4 and SSP5 show the highest difference and SSP2 and SSP3 the lowest. Different to the urban and the coastal approach, the dynamic approach shows a reduced exposure for SSP3 and a higher difference for SSP2 than for SSP4 for 2090 to 2100, when the basic approach is used as reference. Again, this observation highlights the significance of urbanisation, coastal migration and urban sprawl.. As cities are concentrated in coastal areas, the overall population growth in coastal areas is higher than the national average (represented by the basic approach).

\subsection{Regional}

In this section we focus on the comparison between the basic and the coastal approach, as the coastal approach explicitly considers coastal migration. Results for the dynamic and urban approach on regional level can be found in the SM.

Different to the global patterns, Europe, Northern America and Oceania face the highest exposure under SSP5 for both coastal and basic SSPs (Fig. 3). Exposure increases continuously until 2100 under this SSP. For Africa and Latin America and the Caribbean (LAatC), SSP3 shows the highest exposure throughout the century, which also increases continuously with time. This is in line with the underlying national projections of KC and Lutz (2017) that project highest population under SSP5 for the most developed countries and under SSP3 for developing countries. For Asia, we find a notable difference between the coastal and basic approach. In the basic approach, exposure is highest under SSP3 throughout the $21^{\text {st }}$ century. In the coastal approach, exposure is highest under SSP5 until 2075 and under SSP3 afterwards. Asia's high exposure under SSP5 in the coastal approach reflects the high increase of urbanisation levels in the underlying urbanisation projections (Jiang and O'Neill 2017) and the coastward migration in the coastal SSPs. The decrease in Asia's exposure projected after 2050 is due to the decreasing population after 2050 in the underlying population projections (KC and Lutz 2017). This also can also be seen in the basic approach and holds true for all SSPs except SSP3, where the Asia's population is projected to grow after 2050 . 

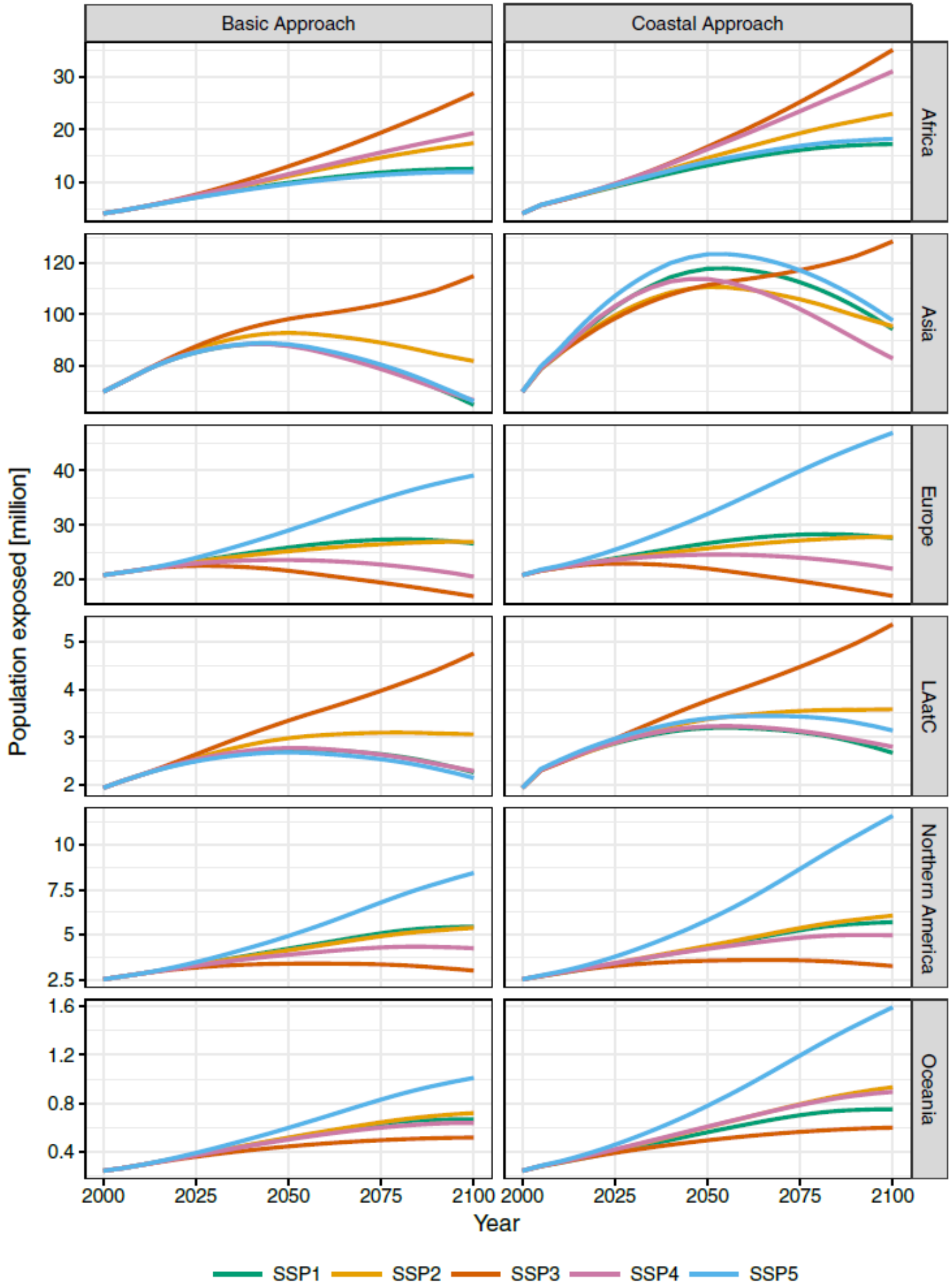

Fig. 3: Exposure per continent under medium SLR in RCP6.0. In the basic approach, SSP1 and SSP5 overlap for Africa and Asia. For LAtaC (Latin America and the Caribbean), SSP1 and SSP4 overlap in both approaches.

The absolute difference in exposure to 1 in 100-year coastal floods on continental scale follows global patterns and becomes larger with SLR in all SSPs (see Fig. A. 7). Accordingly, we find the highest differences in RCP8.5 
with high SLR and the smallest differences in RCP2.6 with low SLR. The difference between the coastal and basic approach is highest in SSP5 in all continents except Africa, where SSP4 shows the highest differences. We observe the lowest differences in SSP1 for Africa, LAatC, Northern America and Oceania. For Europe and Asia, we find the lowest differences between the coastal and basic approach in SSP3.

The relative difference in exposed population is heterogeneous and does not follow the global patterns. For Africa, which shows overall the highest values, we find the relative difference to decrease with rising sea levels (see Table A. 1). The highest difference in exposure is in SSP4 (coastal approach is up to 64\% higher than the basic approach) and lowest in SSP2 and SSP3 (coastal approach 24\% higher than basic approach). For Asia, we find the highest relative differences between coastal and basic approach in SSP5 (48\%) and the lowest in SSP3 ( 12\%). For Europe, which shows overall the closest agreement between coastal and basic approach, the relative difference in exposure increases slightly with SLR. SSP5 exhibits the highest relative difference in exposure ( 20\%) and SSP3 the lowest $(<1 \%)$. For Northern America, the relative difference in exposure increases with SLR in SSP1 and decreases in SSP2-5 while the opposite is the case in Asia. For LAatC and Oceania we do not find a relation between SLR and relative difference in exposure based on the basic and coastal SSPs.

\section{Discussion}

One of our key findings is that under all scenarios the coastal approach projects higher population located in the floodplain of 1 in 100-year coastal floods than the basic approach. In agreement with previous studies that identified urbanisation as a key component in coastal population development, we explain most of the differences with the projected growing urbanisation levels in the coastal approach (see Fig. A. 1 and Fig. A. 2). Coastal areas today show a higher concentration of cities than inland areas. Kummu et al. (2016) shows that 105 out of 256 cities with a population of more than 1 million are located in the near coast zone (proximity to coast $<100 \mathrm{~km}$ and altitude $<100 \mathrm{~m}$ ). According to Brown et al. (2013), in 201020 out of 31 megacities (cities with more than 8 million inhabitants) were located in the low-elevation coastal zone (LECZ; altitude $\leq 10 \mathrm{~m}$ and hydrological connection to the ocean). Neumann et al. (2015) assume that the number of megacities in the LECZ will increase to 25 until 2025. Hoornweg and Pope (2016) project the population development of the 101 largest cities under three SSPs. They show that the percentage of population living in these cities will increase from $11 \%$ in 2010 to $15 \%$ in SSP3, $20 \%$ in SSP2 and $23 \%$ in SSP1 until 2100. As the coastal approach accounts for urbanisation (Merkens et al. 2016) and the basic approach does not, coastal population tends to be underestimated in the basic approach. 
The basic approach shows similar results to the study of Jongman et al. (2012) that also used a homogeneous population growth approach on national level. They found an increase in population exposure to 1 in 100 -year coastal floods between 2010 and 2050 of $25 \%$ on global scale. In the basic approach, we find an increase of population's exposure to 1 in 100-year coastal floods between 19\% in SSP4 and 28\% in SSP3. The exposure based on the coastal approach grows from 2010 to 2050 between 33\% in SSP3 and 50\% in SSP5 and exceeds the projections of Jongman et al. (2012). In agreement with Jongman et al. (2012), both approaches analysed in context of this paper project the highest absolute growth in exposed population until 2050 for Asia and the highest relative growth for Africa. However, the comparison of results to other studies proofs difficult, as the underlying population projections are different. For example, Jongman et al. (2012) used the medium Fertility projection of the 2006 Revision by the UN Population Division while this study is based on the work of KC and Lutz (2017).

The differences in population exposure between the approaches for the years 2005 and 2010 are due to using differing definitions of 'urban' in the underlying data. The urbanisation projections rely on Jiang and O'Neill (2017), which used the world urbanisation prospects (UN 2015) as input data that retains the urban definitions used by each country. Across countries, the definitions are inconsistent. The coastal SSPs of Merkens et al. (2016) used the GRUMP urban extents grid, which tends to underestimate urban extents in developing regions (see section 2.1). Hence, urban population is concentrated in the remaining settlements with night-lights, leading to higher estimated population counts in these areas. As coastal areas in eastern and northern Africa are heavily populated (Hinkel et al. 2012) and western Africa hosts important port cities with growing population (Hanson et al. 2011), the inconsistencies in data trigger an offset in the initial exposure. In SSP4, which shows the highest relative differences between the coastal and basic approach for Africa, the African population grows more than threefold (KC and Lutz 2017) and the urbanisation level almost doubles until 2100 (Jiang and O’Neill 2017). This leads presumably to an overestimation of exposure in the coastal approach. With SLR, the effects of the initial inconsistencies in the data decrease, leading to a reduction of the relative differences of exposed population.

This study has focused on the differences in exposure that arise from using different approaches to regionalise population projections. We interpret the differences in exposure between the approaches as uncertainty that is related to regionalisation, as the underlying population projections on national level do not differ between the approaches. Other uncertainties arise from elevation data and the base year population datasets used to assess the exposure to 1 in 100-year. Elevation and population datasets can potentially be improved if data availability improves and the need for modelling decreases. The uncertainties that arise from the downscaling approach can be reduced to some extent, if the differences between reported urbanisation level and the urbanisation levels based 
on remote sensing products find a better agreement. Other parts of the uncertainty cannot be removed, as the projections are made for long timeframes and human behaviour cannot be predicted.

\section{Conclusion}

This study compared different approaches to account for population change in coastal impact assessment in order to assess the exposure of population to 1 in 100-year coastal floods under different SLR and socioeconomic scenarios. All approaches were based on the same population projections on national level. We found that urbanisation and coastal migration lead to increased exposure whereas urban sprawl leads to reduced exposure. This emphasises the need for taking into account population dynamics on subnational level in exposure assessments. We believe that the exposure estimates obtained from approaches accounting for regional variations in population distribution, such as urbanisation, coastal migration and urban sprawl, are more reliable than the approaches not accounting for such variations. As coastal areas host a disproportionately large number of cities, sub-national population dynamics are of particular relevance for coastal exposure studies and should not be ignored. With rapidly growing cities in developing countries, the need to provide improved assessments of population exposure to coastal flooding is important for global and national planning, both in terms of allocating human and financial resources on national level and climate change adaptation funding on international level.

Acknowledgments: The authors would like to express heir thanks to the editor and the reviewers for their valuable comments. We also would like to thank Maureen Tsakiris for illustrating Fig. 1.

\section{References}

Arnell NW, Livermore M, Kovats S, Levy PE, Nicholls R, Parry ML, Gaffin SR (2004) Climate and socio-economic scenarios for global-scale climate change impacts assessments: Characterising the SRES storylines. Global Environmental Change 14(1):3-20

Arns A, Dangendorf S, Jensen J, Talke S, Bender J, Pattiaratchi C (2017) Sea-level rise induced amplification of coastal protection design heights. Scientific reports 7:40171

Balk DL, Deichmann U, Yetman G, Pozzi F, Hay SI, Nelson A (2006) Determining Global Population Distribution: Methods, Applications and Data. In: Meltzer MI (ed) Global Mapping of Infectious Diseases: Methods, Examples and Emerging Applications, vol 62. Advances in Parasitology. Elsevier, pp 119-156 
Brown S, Nicholls RJ, Woodroffe CD, Hanson S, Hinkel J, Kebede AS, Neumann B, Vafeidis AT (2013) Sea-Level Rise Impacts and Responses: A Global Perspective. In: Finkl CW (ed) Coastal Hazards, vol 1000. Coastal Research Library. Springer Netherlands, Dordrecht, pp 117-149

Church JA, Clark PU, Cazenave A, Gregory JM, Jevrejeva S, Levermann A, Merrifield MA, Milne, GA, Nerem RS, Nunn PD, Payne AJ, Pfeffer WT, Stammer D, Unnikrishnan AS (2013) Sea Level Change. In: Stocker TF, Qin D, Plattner G-K, Tignor M, Allen S, Boschung J, Nauels A, Xia Y, Bex V, Midgley P (eds) Climate Change 2013: The Physical Science Basis: Contribution of Working Group I to the Fifth Assessment Report of the Intergovernmental Panel on Climate Change. Cambridge University Press, Cambridge, pp 1137-1216

CIESIN (Center for International Earth Science Information Network - Columbia University), IFPRI (International Food Policy Research Institute), (The World Bank), CIAT (Centro Internacional de Agricultura Tropical) (2011a) Global Rural-Urban Mapping Project, Version 1 (GRUMPv1): Population Count Grid. NASA Socioeconomic Data and Applications Center (SEDAC), Palisades, NY

CIESIN (Center for International Earth Science Information Network - Columbia University), IFPRI (International Food Policy Research Institute), (The World Bank), CIAT (Centro Internacional de Agricultura Tropical) (2011b) Global Rural-Urban Mapping Project, Version 1 (GRUMPv1): Urban Extents Grid. NASA Socioeconomic Data and Applications Center (SEDAC), Palisades, NY

Engström K, Olin S, Rounsevell MDA, Brogaard S, van Vuuren DP, Alexander P, Murray-Rust D, Arneth A (2016) Assessing uncertainties in global cropland futures using a conditional probabilistic modelling framework. Earth Syst. Dynam. 7(4):893-915

Gaffin SR, Rosenzweig C, Xing X, Yetman G (2004) Downscaling and geo-spatial gridding of socioeconomic projections from the IPCC Special Report on Emissions Scenarios (SRES). Global Environmental Change 14(2):105-123

Gao J, (2017) Downscaling Global Spatial Projections from 1/8-degree to 1-km Grid Cells. NCAR Technical Note NCAR/TN-537+STR 
Grübler A, O'Neill B, Riahi K, Chirkov V, Goujon A, Kolp P, Prommer I, Scherbov S, Slentoe E (2007)

Regional, national, and spatially explicit scenarios of demographic and economic change based on SRES. Technological Forecasting and Social Change 74(7):980-1029

Hallegatte S, Green C, Nicholls RJ, Corfee-Morlot J (2013) Future flood losses in major coastal cities. Nature Climate change 3(9):802-806

Hanson S, Nicholls R, Ranger N, Hallegatte S, Corfee-Morlot J, Herweijer C, Chateau J (2011) A global ranking of port cities with high exposure to climate extremes. Climatic Change 104(1):89-111

Hinkel J, Brown S, Exner L, Nicholls RJ, Vafeidis AT, Kebede AS (2012) Sea-level rise impacts on Africa and the effects of mitigation and adaptation: an application of DIVA. Reg Environ Change 12(1):207-224

Hinkel J, Lincke D, Vafeidis AT, Perrette M, Nicholls RJ, Tol, Richard S. J., Marzeion B, Fettweis X, Ionescu C, Levermann A (2014) Coastal flood damage and adaptation costs under 21st century sealevel rise. Proc Natl Acad Sci USA 111(9):3292-3297

Hinkel J, Nicholls RJ, Tol RS, Wang ZB, Hamilton JM, Boot G, Vafeidis AT, McFadden L, Ganopolski A, Klein RJ (2013) A global analysis of erosion of sandy beaches and sea-level rise: An application of DIVA. Global and Planetary Change 111:150-158

Hinkel J, Nicholls RJ, Vafeidis AT, Tol RS, Avagianou T (2010) Assessing risk of and adaptation to sea-level rise in the European Union: an application of DIVA. Mitig Adapt Strateg Glob Change 15(7):703-719

Hoornweg D, Pope K (2016) Population predictions for the worlds largest cities in the 21st century. Environment and Urbanization

Jarvis A, Reuter HI, Nelson A, Guevara E (2008) Hole-filled SRTM for the globe Version 4: Available from the CGIAR-CSI SRTM 90m Database. http://srtm.csi.cgiar.org

Jiang L, O’Neill BC (2017) Global urbanization projections for the Shared Socioeconomic Pathways. Global Environmental Change 42:193-199

Jones B, O’Neill BC (2016) Spatially explicit global population scenarios consistent with the Shared Socioeconomic Pathways. Environ. Res. Lett. 11(8):84003 
Jongman B, Ward PJ, Aerts JC (2012) Global exposure to river and coastal flooding: Long term trends and changes. Global Environmental Change 22(4):823-835

KC S, Lutz W (2017) The human core of the shared socioeconomic pathways: Population scenarios by age, sex and level of education for all countries to 2100. Global Environmental Change 42:181-192

Kummu M, Moel H de, Salvucci G, Viviroli D, Ward PJ, Varis O (2016) Over the hills and further away from coast: Global geospatial patterns of human and environment over the 20th-21st centuries. Environ. Res. Lett. 11(3):34010

Lichter M, Vafeidis AT, Nicholls RJ, Kaiser G (2011) Exploring Data-Related Uncertainties in Analyses of Land Area and Population in the "Low-Elevation Coastal Zone" (LECZ). Journal of Coastal Research 274:757-768

McGranahan G, Balk D, Anderson B (2007) The rising tide: Assessing the risks of climate change and human settlements in low elevation coastal zones. Environment and Urbanization 19(1):17-37

Merkens J-L, Reimann L, Hinkel J, Vafeidis AT (2016) Gridded population projections for the coastal zone under the Shared Socioeconomic Pathways. Global and Planetary Change 145:57-66

Merkens J-L, Vafeidis A (2018) Using Information on Settlement Patterns to Improve the Spatial Distribution of Population in Coastal Impact Assessments. Sustainability 10(9):3170

Mondal P, Tatem AJ (2012) Uncertainties in measuring populations potentially impacted by sea level rise and coastal flooding. PLoS ONE 7(10):e48191

Muis S, Verlaan M, Winsemius HC, Aerts JCJH, Ward PJ (2016) A global reanalysis of storm surges and extreme sea levels. Nature communications 7:11969

Muis S, Verlaan M, Nicholls RJ, Brown S, Hinkel J, Lincke D, Vafeidis AT, Scussolini P, Winsemius HC, Ward PJ (2017) A comparison of two global datasets of extreme sea levels and resulting flood exposure. Earth's Future 5(4):379-392

Neumann B, Vafeidis AT, Zimmermann J, Nicholls RJ (2015) Future coastal population growth and exposure to sea-level rise and coastal flooding--a global assessment. PLoS ONE 10(3):e0118571

Nicholls RJ (2004) Coastal flooding and wetland loss in the 21st century: Changes under the SRES climate and socio-economic scenarios. Global Environmental Change 14(1):69-86 
Nicholls RJ, Mimura N (1998) Regional issues raised by sea-level rise and their policy implications. Climate Resarch 11:5-18

Nicholls RJ, Wong PP, Burkett V, Woodroffe CD, Hay J (2008) Climate change and coastal vulnerability assessment: Scenarios for integrated assessment. Sustain Sci 3(1):89-102

O’Neill BC, Kriegler E, Ebi KL, Kemp-Benedict E, Riahi K, Rothman DS, van Ruijven, van Vuuren DP, Birkmann J, Kok K, Levy M, Solecki W (2017) The roads ahead: Narratives for shared socioeconomic pathways describing world futures in the 21st century. Global Environmental Change 42:169-180

Poulter B, Halpin PN (2008) Raster modelling of coastal flooding from sea-level rise. International Journal of Geographical Information Science 22(2):167-182

Prather M, Flato G, Friedlingstein P, Jones C, Lamarque J-F, Liao H, Rasch P (2014) Annex II: Climate System Scenario Tables. In: Stocker TF, Qin D, Plattner G-K, Tignor M, Allen S, Boschung J, Nauels A, Xia Y, Bex V, Midgley P (eds) Climate Change 2013: The Physical Science Basis: Contribution of Working Group I to the Fifth Assessment Report of the Intergovernmental Panel on Climate Change. Cambridge University Press, Cambridge

Seto KC (2011) Exploring the dynamics of migration to mega-delta cities in Asia and Africa: Contemporary drivers and future scenarios. Global Environmental Change 21:S94-S107

Spencer T, Schuerch M, Nicholls RJ, Hinkel J, Lincke D, Vafeidis AT, Reef R, McFadden L, Brown S (2016) Global coastal wetland change under sea-level rise and related stresses: The DIVA Wetland Change Model. Global and Planetary Change 139:15-30

UN (United Nations, Department of Economic and Social Affairs, Population Division) (2015) World urbanization prospects: The 2014 revision. United Nations, New York

USGS (United States Geological Survey) (1996) Global Digital Elevation Model: GTOPO30. http://earthexplorer.usgs.gov/

van Vuuren DP, Kriegler E, O’Neill BC, Ebi KL, Riahi K, Carter TR, Edmonds J, Hallegatte S, Kram T, Mathur R, Winkler H (2014) A new scenario framework for Climate Change Research: scenario matrix architecture. Climatic Change 122(3):373-386 

Syst. Sci. 16(8):1841-1853 